\title{
Errores en el aprendizaje de integrales a nivel de la Educación Preuniversitaria
}

\author{
Errors in learning integrals at the level of pre-University Education
}

Recibido octubre 2019

Arbitrado noviembre 2019

Publicado enero 2020

\section{Helys Joel Terán Terán \\ helysteran@gmail.com \\ Código ORCID: 0000-0002-9831-1952}

Universidad Nacional Experimental de la Fuerza Armada, Venezuela

\section{Resumen}

El objetivo de esta investigación fue analizar los errores en el aprendizaje de matemática preuniversitaria, que presentan los estudiantes de Economía Social, en la resolución de integrales de la asignatura Matemática II de la Universidad Nacional Experimental Politécnica de las Fuerzas Armadas. Se fundamentó en la Tipología de Astolfi. La investigación fue de campo no experimental, nivel descriptivo. Los datos se obtuvieron a través de pruebas de conocimiento aplicadas durante el semestre académico, la muestra estuvo conformada por trece participantes. El instrumento fue sometido al juicio de expertos para su validación, la confiabilidad se obtuvo por medio del coeficiente de correlación Pearson. Se concluyó que los estudiantes no están debidamente preparados con los contenidos previos para desenvolverse satisfactoriamente. Se recomienda a los docentes que identifiquen los errores que presentan sus estudiantes de manera que a éstos se les dé un nuevo enfoque, convertirlos mediante novedosas estrategias, en una oportunidad para aprender.
Palabras clave:

Errores;

Matemática preuniversitaria; Aprendizaje
The objective of this investigation was to analyze the errors in the learning of pre-university mathematics, presented by the students of Social Economy, in the resolution of integrals of the subject Mathematics II of the National Experimental Polytechnic University of the Armed Forces. It was based on the Astolfi Typology. The research was non-experimental field, descriptive level. The data was obtained through knowledge tests applied during the academic semester, the sample was made up of thirteen participants. The instrument was subjected to expert judgment for validation, reliability was obtained using the Pearson correlation coefficient. It was concluded, the students are not adequately prepared with the previous contents to perform satisfactorily. Teachers are recommended to identify the errors presented by their students so that they are given a new approach, turning them through innovative strategies, into an opportunity to learn.

\author{
Keywords: \\ Errors; \\ Pre-university \\ mathematics; \\ Learning
}




\section{INTRODUCCIÓN}

$\mathrm{E}$ l error forma parte de la cotidianidad del ser humano, pues podría decirse que es un conocimiento deficiente e incompleto en cualquier faceta de su vida. El campo educativo no escapa de ello; en el proceso de la enseñanza y aprendizaje de la matemática, siempre ha existido, en todos los niveles educativos, la preocupación por los errores de aprendizaje presentes en el desempeño del alumnado, ya que éstos representan uno de los factores que lo inducen al fracaso escolar. Prueba de esta preocupación se traduce en las múltiples investigaciones, que han surgido al respecto, en todos los ámbitos educativos y en muchas partes del mundo.

Es importante tomar en consideración que el error en el aprendizaje de la matemática debería asumirse, no como un elemento que está allí presente, sino, como un elemento que pueda ser útil e interesante para la adquisición y reformulación de un nuevo y mejor conocimiento. Ahora bien, el error se manifiesta en el estudiante a través de su capacidad cognitiva, siendo más preciso, en las operaciones matemáticas, puesto que al desarrollar las actividades de matemática se observan constantes errores, según lo expresado por De la Torre (2004) "el alumno realiza las operaciones matemáticas de forma mecánica, porque ya las domina, pero ante el error cometido prestar mayor atención para descubrir dónde pudo tener el fallo" (p. 49).

De acuerdo al autor antes mencionado, el estudiante incurre en el error al realizar las operaciones matemáticas de manera mecánica, debido a que olvidan realizar un análisis cuidadoso antes de dar respuesta, sin embargo, al cometer un error y hacerlo consciente está dispuesto en aclarar las dificultades que se le presentan. No sólo existe preocupación a nivel del profesorado por este tema, además, existen organizaciones que se interesan en realizar estudios mediante programas con la finalidad de conocer el nivel de desempeño matemático, tal es el caso.

Es por ello que, al observar de manera general los diferentes niveles de la educación Venezolana, se puede notar que los educandos no escapan de estas dificultades y pareciera tener mayor incidencia en el área de la matemática, por lo cual es importante escudriñar en el proceso de aprendizaje de los estudiantes. Ante lo expuesto, es válido considerar que todo docente al entrar en un aula 
de clases puede percibir, a través de diferentes formas de expresión, las expectativas, metas u objetivos de los educandos con respecto a la materia, sin embargo, lo que es difícil de notar a primera vista es la manera en la cual los estudiantes procesan la información que se les emite, ya que cada uno es diferente y tiene una forma especial de organizar sus ideas y construir su propio conocimiento.

En este sentido, Popper y Lorenz (2000) afirman que: "nuestra cabeza es un cubo con una tapa llena de agujeros, a través de los cuales se infiltra información procedente del mundo. Esa es la teoría que fundamenta la pedagogía actual" (p.70). Esta perspectiva del autor, sugiere que toda la información que se nos suministra es depositada en la cabeza, sin embargo no detalla cómo se procesa, pues cada individuo es único y diferente. Tal como lo expresa, Briceño (2011), al referirse que el aprendizaje:

(...) parte siempre de la recepción de algún tipo de información, de todos los datos que se reciben, se selecciona y se organiza la información. Sin embargo, este proceso de aprendizaje es algo más complejo debido a la multiplicidad de variables, elementos y estrategias que influyen en el mismo. En este desarrollo cognitivo están no solamente el recibir y enviar mensajes o contenidos sino también la práctica, análisis, reflexión y razonamiento de pensamientos, ideas y estructuras. Igualmente, no todas las personas aprenden igual ni a la misma velocidad, lo cual no es ninguna novedad. (p.143)

En relación al párrafo antes citado, la autora describe que el aprendizaje parte de los datos obtenidos, los cuales deben procesarse mediante la organización de la información, pero que no se queda sólo en ello pues existen diversos elementos que influyen en él, de igual manera, hace mención a la importancia que tienen factores como: la práctica, el análisis, reflexión, razonamientos, ideas y estructuras para el desarrollo cognitivo del individuo, por lo cual es importante que el educando estimule su aprendizaje para que éste sea más significativo, atendiendo a su propio estilo, método o estrategia para aprender; es decir, los estudiantes pueden responder de diferentes maneras a la información que recopilan en un aula de clases, tomando en cuenta su propia manera de organizar datos o información. 
Si bien es cierto, cada estudiante es responsable de conocer y familiarizarse con el método, estrategia o estilo que le ayude a profundizar en su propio aprendizaje, también lo es que los educadores son parte fundamental para impulsar y motivar a los estudiantes a descubrir la manera en que éstos pueden mejorar sus conocimientos, es por ello que se toma en consideración la opinión de Briceño (2011), quien manifiesta:

Los estilos y estrategias de aprendizaje sobresalen entre las variables más importantes que influyen en la actuación de los estudiantes, por lo tanto, los facilitadores pueden ayudar a sus estudiantes concibiendo una instrucción que responda a las necesidades de la persona con diferentes preferencias estilísticas y enseñándoles a la vez la forma de mejorar sus estrategias para adquirir conocimiento. (p.145)

Del párrafo descrito anteriormente, vale la pena preguntarse si en el proceso de aprendizaje de la matemática y la minimización de los errores, ¿es importante la didáctica del docente?, citando a D’Amore (2005): “la suposición más o menos explícita parecía ser la siguiente: si se mejora la enseñanza, mejorará también el aprendizaje" (p. 11), en consecuencia, se debe a través de la didáctica de la matemática empleada por el docente, identificar los errores cometidos por el estudiante, los cuales, no se deben asumir como elementos negativos, sino al contrario, tomar acciones de corrección que permitan construir un aprendizaje más significativo.

Podría decirse entonces que estos errores detectados en el aprendizaje del educando pueden mejorarse empleando una didáctica donde no sólo, el horizonte, sea impartir un contenido a cabalidad, sino que es necesario interesarse también en cómo va a ser enseñado ya que es importante tomar conciencia en lo que se refiere a la necesidad de perfeccionar la didáctica en beneficio de la formación del estudiante.

De tal manera que, el docente de matemática debe prestar atención al identificar errores en el aprendizaje de los estudiantes, debido a que es él quien facilita los elementos de aprendizaje a través de la enseñanza, ya que debería estar en constante actualización e intercambio de opiniones en conferencias sobre didáctica de la matemática y pedagogía en cuanto a la educación, al respecto Mora (2004) señala: 
La complejidad que caracteriza la educación, está determinada por un conjunto de variables pedagógicas, didácticas, culturales, sociales, económicas y políticas, lo cual compromete continuar más en la búsqueda de caminos con la finalidad de mejorar la práctica como docentes de aula y, por lo tanto, aportar argumentos que impulsen la transformación de la educación matemática. (p. 84)

Como consecuencia de esto, se podría obtener una mejora en la formación de los aprendices y así disminuir los errores y obstáculos que no los dejan avanzar; sin embargo, pareciera que el estudiante en su proceso de aprendizaje pasa por alto estos elementos o estrategias didácticas que el docente utiliza para obtener resultados favorables en el proceso de enseñanza y aprendizaje de la matemática.

Además, para la obtención de resultados favorables en matemática, se debe tomar en consideración al ser humano en todas sus etapas y pasos por los diferentes niveles del sistema educativo; en tal sentido, el niño desde su primer contacto con el mundo que lo rodea, va asimilando de una manera informal la idea de número, forma, tamaño y en su evolución va incorporando nuevos contenidos, de allí que la matemática siempre se encuentra inmersa en las actividades del diario vivir, es así como el niño explora y usa la lógica racional, como característica innata va desarrollando la disciplina matemática con la estimulación de padres, como primeros maestros y el medio ambiente; de acuerdo al boletín emitido por Massachusetts PIRC.

Luego de este aprendizaje intuitivo, el niño comienza su tránsito hacia un aprendizaje formal. En este camino hacia el aprendizaje de la matemática, el niño va concibiendo conceptos o procedimientos erróneos que se convierten en preocupación constante para el docente, ya que éstos aparecen de manera sistemática en la construcción del conocimiento, por tal motivo, en este proceso debería estar presente la corrección y superación de los mismos mediante actividades que le permitan al estudiante tener una práctica constante, y así disminuir las dificultades presentes en el procedimiento y manejo adecuado de la definición de los contenidos matemáticos.

Es así que el docente, en los primeros años de formación del niño específicamente en la Educación Básica, juega un papel fundamental, debido a que si el niño en esta etapa comete errores y 
se penaliza como un acto negativo, podrían convertirse estas dificultades en una forma de odio o rechazo, lo que en un futuro pudiera transformarse en uno de los factores de retraso académico y quizás de deserción escolar y exclusión social, esto sustentado en un artículo de Rivas (2005), donde plantea:

Un docente cuya visión del fenómeno educativo se expresa en los hechos, negándole al niño la posibilidad de que construya y reconstruya los saberes escolares, en virtud de asumirse él, como fuente básica del conocimiento, propiciando una nefasta dependencia en el niño y creando condiciones favorables para que el verbalismo, el formulismo y el aprendizaje mecánico se instalen desde temprana edad escolar. (p. 167)

Es decir, si quienes imparten una enseñanza matemática, desde una adición o sustracción, emplean recursos y metodologías pedagógicas que promueven más que técnicas, procedimientos reales, lógicos y contextualizados, adecuados para los primeros años de educación en el proceso de enseñanza y aprendizaje, el proceso sería más efectivo en el individuo en formación, evitando dificultades y errores en el aprendizaje del educando.

El perfil del docente, requiere de acciones concretas, que sin duda se relacionan con el profesional que ejerce en el campo de la matemática, es por ello que ser docente no significa vaciar contenidos repetitivos, definitivos, es necesario que se aborden con propiedad nuevos paradigmas, en miras hacia una educación de calidad, en este sentido Murillo (2003) afirma que el docente necesita: "una interesante propuesta de actualización del maestro de matemática bajo los nuevos preceptos teóricos-prácticos de las matemáticas a través de situaciones de aprendizajes significativos tomadas de la vida cotidiana" (p. 178). De acuerdo a esto, se vislumbra que en la matemática es propicio incorporar diversas situaciones de aprendizaje que permiten generar en los educandos un aprendizaje significativo, que atienda a su contexto cotidiano, promoviendo así un conocimiento funcional en el estudiante, que permita aportar soluciones en el medio que le rodea.

En los párrafos anteriores, se ha insistido en la preparación del docente, ya que debe ir a la par con la enseñanza, y todo buen método de aprendizaje debe incidir en el estudiante asertivamente, adecuar diversas estrategias para la mediación de los contenidos que 
permitirán el logro de los objetivos planteados. El Currículo Básico Nacional y sus programas presentan nuevos modelos contextualizados y articulados con otras asignaturas para la enseñanza y aprendizaje de la matemática, dándole un carácter de interdisciplinario, pero se desconocen los resultados de investigaciones al respecto y se evidencia rendimiento poco satisfactorio en carreras universitarias, donde el conocimiento previo, matemática adquirida en niveles anteriores, se es requerido.

En lo que se refiere al Currículo Nacional Bolivariano, del subsistema de Educación Primaria que contempla lo siguiente: "el maestro y la maestra planificarán junto con los niños, las niñas y otros colegas, las experiencias de aprendizajes que se caractericen por la investigación y que conlleven tanto a la comprensión de ideas matemáticas, como estrechar relaciones con el ambiente" (p. 21), de esta manera los docentes en conjunto con los niños y niñas se apropian de los paradigmas innovadores, entonces cabría preguntarse: ¿En la realidad se aprecia y practica lo descrito en éste y en los párrafos anteriores?, ¿el maestro le da sentido a esta contextualización?, ¿̇ha sido o se ha preparado para ello?

En virtud de esto último, haciendo una reflexión en la manera común de enseñar matemática y, de generar aprendizajes en los distintos niveles del sistema educativo, particularmente, en los primeros grados básicos de la educación integral del educando, la pedagogía de la escuela pareciera que produce el conjunto de creencias o mitos de la traumática experiencia de muchas generaciones de estudiantes en cuya memoria escolar está anidada el estigma de una matemática que despertó miedo en su edad escolar.

Por otro lado y tomando en cuenta las consideraciones anteriores, en las instituciones de Educación Media General, a pesar de que en la educación matemática hay docentes que toman posturas tradicionalistas, otros modernas y avanzadas, existen muchos que tienen el propósito de ayudar a superar los errores que presentan los estudiantes en su aprendizaje, con el objetivo de eliminar las costumbres escolares tradicionalistas donde sólo se dedica a recibir contenidos que a la larga inducen hacia el error en el aprendizaje de la matemática.

En otro orden de ideas, las demandas que surgen en este contexto educativo correspondiente al nivel superior ya no son solamente lograr los aprendizajes tradicionales de la escuela sino 
manejar e interpretar contenidos y estrategias didácticas que permitan un aprendizaje significativo y con él, desarrollar un conocimiento apto que le permita al educando desenvolverse en un futuro en un campo profesional.

En este mismo sentido, es importante resaltar que las instituciones de educación superior tienen un papel fundamental en la formación de la ciudadanía, es decir los docentes de matemática en educación superior deben contar con una preparación que les permita detectar y diagnosticar los errores que el estudiante trae de niveles previos, además adaptar estrategias metodológicas que ayuden a superar dichos errores.

Ahora bien, si el docente de matemática de educación superior debe subsanar los errores, se hace cuesta arriba el cumplimiento de los objetivos propuestos en este nivel. En tal sentido, es necesario el estudio de errores de aprendizaje que están de forma constante en las evaluaciones aplicadas a los estudiantes, por lo que amerita hacer un análisis de los mismos para contribuir a minimizarlos, de tal manera, que no interfiera en el logro de los nuevos objetivos. Por ende, los errores que padecen los estudiantes en el nivel de educación superior, deberían ser tomados en cuenta, revisados y analizados para minimizarlos, en este sentido, Socas (2007) plantea lo siguiente:

Las investigaciones desarrolladas en este sentido nos han llevado a profundizar más en el origen y causa de los errores y a revisar los errores desde dos puntos de vista: las dificultades inherentes a las Matemáticas y las dificultades inherentes al proceso de enseñanza y aprendizaje de las mismas en el ámbito escolar. (p. 24)

Entonces se puede decir que las dificultades del estudiantado por constantes errores en el aprendizaje de la matemática, es lo que ha motivado el presente estudio, donde el investigador, a través de su experiencia como docente, ha venido observando, con preocupación, en la Universidad Nacional Experimental Politécnica de las Fuerzas Armadas (UNEFA), extensión del núcleo de Carabobo ubicada en el Municipio Guacara del Estado Carabobo; el desempeño poco satisfactorio de los estudiantes en la asignatura Matemática II, de la carrera de Economía Social, y al parecer, una de las causas del problema está en concepciones erróneas y la falta de los conocimientos previos en el aprendizaje de la matemática de niveles 
anteriores, matemática preuniversitaria, los cuales se traducen en altos porcentajes de aplazados en los parciales de dicha asignatura, sobre todo al momento de resolver integrales, estando este contenido presente en todos los parciales.

Todo esto, vinculado con las observaciones del investigador en el segundo semestre del año 2018, le llevan a plantearse las siguientes interrogantes: ¿Cuáles son los errores en el aprendizaje de matemática preuniversitaria que presentan los estudiantes cursantes de la asignatura Matemática II? ¿Qué errores presentan los estudiantes en la resolución de las integrales propuestas en los parciales respectivos de la asignatura matemática II?, ¿De qué manera los errores en el aprendizaje de matemática preuniversitaria están presentes en el desempeño de la asignatura Matemática II de Economía Social?

Por esta razón se fijó como objetivo general analizar los errores en el aprendizaje de matemática preuniversitaria, que presentan los estudiantes en la resolución de integrales, de la asignatura Matemática II de Economía Social de la Universidad Nacional Experimental Politécnica de las Fuerzas Armadas (UNEFA), extensión Guacara del Estado Carabobo.

Para ello, se hizo necesario en primer lugar identificar los tipos de errores en el aprendizaje de matemática preuniversitaria, posteriormente determinar los errores de aprendizaje presentes en las evaluaciones parciales de la asignatura Matemática II; y así describir los tipos de errores, que afectan la correcta solución de las integrales, en las evaluaciones parciales de la asignatura Matemática II de la carrera Economía Social.

Ahora bien, es de hacer notar, la matemática contempla una de las áreas del conocimiento indispensable para el desarrollo del pensamiento en el ser humano, ella vislumbra procesos racionales que ayudan y promueven la cognición, mediante la comprensión y análisis de situaciones, de tal manera que el individuo interactúa diariamente con los elementos que en ella intervienen, a su vez el estudio de errores cometidos por los estudiantes se convierte en una de las actividades de la acción educativa, es por ello que el presente estudio está orientado al análisis de errores de aprendizaje que traen consigo los estudiantes de la educación matemática preuniversitaria y su permanencia en matemática de educación universitaria. Al respecto la opinión de Morín (2003), constituye un aporte de gran relevancia, ya que afirma: 
La racionalidad puede ser definida como un conjunto de las cualidades de verificación, control, coherencia, adecuación, que permiten asegurar la objetividad del mundo exterior y operar la distinción y la distancia entre nosotros y este mundo. A partir de ahí, y visto que todo conocimiento es traducción y reconstrucción y que las fermentaciones fantásticas parasitan cualquier conocimiento, el error y la ilusión son los problemas cognitivos permanentes de la mente humana. A pesar de sus capacidades de control y verificación, el conocimiento humano ha corrido y sigue corriendo riesgos formidables de error (...) (p. 108)

Considerando el planteamiento anterior, es necesario destacar que el error forma parte de la cotidianidad y que es normal en la capacidad humana; sin embargo el hacer conscientes las situaciones donde éste se expone, es probable que disminuya en las futuras ocasiones, pues empleando la racionalidad, el individuo es capaz de actuar con coherencia y reconstruir el conocimiento superando o evitando el error.

En vista de que la racionalidad en el individuo se desarrolla desde el momento de su nacimiento, es relevante mencionar que la matemática es una de las pocas áreas con la cual se trabaja desde la etapa inicial, donde se fundamenta la base del conocimiento, requisito previo para el buen desenvolvimiento en los estudiantes a medida que avanzan; es en este nivel de educación primaria donde se fija lo que será en estudios posteriores el dominio de conceptos y algoritmos utilizados en matemática de bachillerato (matemática preuniversitaria) por lo que, a través de esta investigación se realizan unas pruebas con las cuales se pretende conocer y determinar los errores en el aprendizaje de la matemática que persisten en los estudiantes de las universidades venezolanas, específicamente en la UNEFA.

En este sentido, el presente trabajo de investigación pretendió dar respuesta a las líneas estratégicas del sistema educativo superior, lo que permite promover favorablemente un beneficio académico en los estudiantes en cuanto al aprendizaje para entonces ir en pro de una calidad de educación, es por ello que constantemente al inicio de año o semestre se debe tener como premisa reforzar lo ya adquirido, por lo que el docente debe hacer un diagnóstico para detectar las necesidades que puedan, los estudiantes, presentar en esta etapa preuniversitaria. 
De hecho, se considera que los errores conducen a la frustración, repitencia y deserción del estudiantado de la asignatura Matemática II de Economía Social, por lo que este estudio contribuye de manera significativa a minimizar estos efectos, divulgando los hallazgos de la misma ante los docentes, no sólo universitarios sino de educación media general, a fin de poder discernir en los factores que inciden en esta problemática.

Es por ello, que el presente estudio se interesa en el análisis de errores permanentes que se evidencian en matemática II como consecuencia del desconocimiento de conceptos, procedimientos o poca comprensión por parte de los estudiantes y así mejorar los errores en el aprendizaje de matemática preuniversitaria, su permanencia en matemática II, no sólo brindando un beneficio a los estudiantes y docentes de la institución donde se desarrolló la investigación, sino también a otras instituciones e investigadores que deseen abordar problemáticas con índoles similares.

Por otra parte, esta investigación constituye un punto de partida a otras investigaciones tales como el estudio no solo de errores, sino de dificultades y obstáculos que incidan en el desempeño de los estudiantes, por ende se facilita a otros investigadores el estudio de esta temática ya que es una variante en la educación matemática en el mundo y particularmente en Venezuela. En este sentido se puede aseverar el aporte filosófico, teórico, metodológico, pedagógico y andragógico que el presente estudio manifiesta, pues además de los autores que sustentan la investigación con sus postulados y teorías, se encuentra un tipo de estudio que sugiere una base a futuras investigaciones, de igual manera, a docentes de Educación Media y Universitaria que deseen estudiar y ampliar este tipo de fallas.

\section{MÉTODO}

L a investigación cuantitativa se enmarcó en el paradigma positivista; es por ello que el presente estudio se enmarcó en un diseño no experimental, de campo descriptivo, ya que se diagnosticaron los tipos de errores que presentan los estudiantes en el aprendizaje de sus conocimientos previos de matemáticas y de qué manera, están presentes en el desempeño del estudiantado de la asignatura Matemática II de Economía Social, haciendo una descripción detallada, mediante el registro de los hallazgos 
obtenidos en el estudio realizado interpretando las realidades del hecho.

Para realizar el análisis de errores que cometen los estudiantes con mayor frecuencia en el aprendizaje de la Matemática del nivel de Educación Media General, se realizó una evaluación al inicio del semestre para diagnosticar los conocimientos previos de los estudiantes; así como también, durante el desarrollo del semestre se aplicaron ocho parciales de los cuales se tomaron para el estudio sólo cuatro de la asignatura Matemática II de la UNEFA; de igual manera, para el análisis se tomó como base la fundamentación teórica y los objetivos planteados, se siguieron los procedimientos de investigación que se llevaron a cabo en tres fases. En la descripción, se hace referencia tanto a las técnicas usadas como a la forma en que se aplicaron.

Se confeccionó el instrumento de la evaluación, el cual constó de diez (10) ítem orientados a indagar cuáles eran los conocimientos previos en el aprendizaje de la Matemática en la Educación Media General. Se empleó un lenguaje acorde al nivel en que se encontraban los educandos con el objetivo de que se sintieran cómodos y entendieran el sentido de cada pregunta en la evaluación, la cual se aplicó con la denominación prueba diagnóstico.

Atendiendo a las consideraciones anteriores, se estudió a la población en su totalidad; es decir, significa que se realizó un censo o estudio de tipo censal, la cual estuvo conformada por trece (13) estudiantes, en consecuencia, la cantidad antes descrita de estudiantes fueron quienes se inscribieron y cursaron en el segundo período lectivo del 2018 (II-2018), en la asignatura Matemática II de la carrera Economía Social.

El instrumento antes mencionado, según Palella y Martins (2012): “(...) pueden ser escritas u orales. Generalmente están construidas con preguntas abiertas y requieren la elaboración de respuestas por parte del investigado" (p.145); esta prueba tipo ensayo fue empleada, en un primer momento como prueba diagnóstico, es decir, para recolectar la información necesaria, en cuanto a los conocimientos de matemática preuniversitaria en los estudiantes de Matemática II. La prueba diagnóstico se elaboró, partiendo de los conocimientos básicos que deberían dominar los estudiantes egresados del nivel de Educación Media General y que a la vez son requisitos básicos en la asignatura de Matemática II. 
Para la validez, se empleó la técnica del juicio de experto, ésta consistió en entregar a tres (3) expertos en el área y para determinar la confiabilidad el coeficiente de correlación Pearson correspondiente a las pruebas de formas equivalentes o de formas alternas. El valor arrojado para el coeficiente Pearson fue de 0,75, lo que indica un nivel de correlación positiva considerable.

\section{RESULTADOS Y DISCUSIÓN}

A continuación se examinó si los errores en el aprendizaje de matemática preuniversitaria presentes en los estudiantes de Matemática II de Economía Social, repercutieron en el desempeño de éstos al resolver los integrales en dicha asignatura, es decir, se vislumbra aquí la columna vertebral del presente trabajo de investigación, mostrando los resultados e inquietudes del investigador, en el estudio de las dificultades y errores que se encuentran en la población estudiantil que ingresa a la UNEFA.

En este sentido, se da respuesta a los objetivos propuestos, presentando a continuación los errores encontrados, luego de aplicar un primer instrumento (prueba diagnóstico), con respecto a los conocimientos de matemáticas adquiridos en Educación Media General ya analizados anteriormente, para determinar de esta manera cómo están presentes e influyen estos errores en el desenvolvimiento de la asignatura. Así, se comienza por agrupar los contenidos de matemática preuniversitaria presentes en las evaluaciones de esta asignatura, tomando en cuenta estos temas de estudio, se expresan el número de errores hallados de acuerdo a la tipología de error.

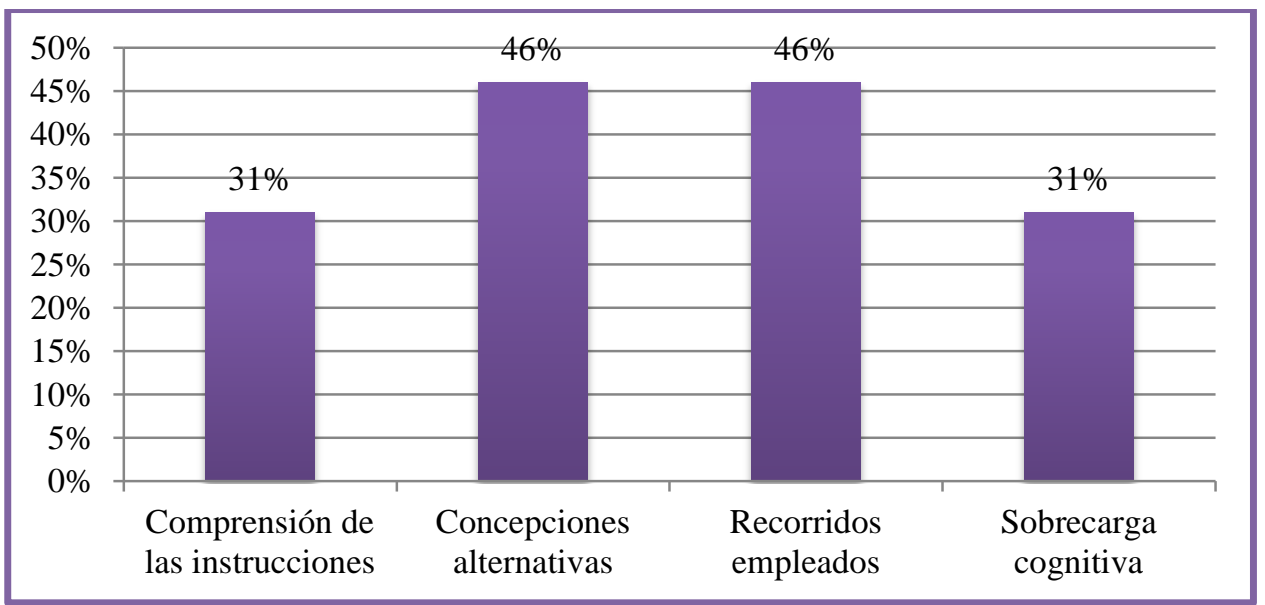

Gráfico 1. Análisis de los errores presentes en el diagnóstico de acuerdo a la tipología de Astolfi (1999). (Fuente: Terán, 2020) 
En este gráfico 1 se representan los porcentajes de frecuencia de acuerdo a los tipos de errores de acuerdo con la tipología de Jean Pierre Astolfi (1999); por lo cual al momento de ir separando la información y analizando la tipología de error, para cada uno de los temas que integraron las evaluaciones, se observó, en el desarrollo de la primera evaluación parcial de Matemática II, los errores más frecuentes devinieron de concepciones alternativas y de recorridos empleados; 6 estudiantes (46\%) antes de resolver los integrales debían desarrollar el producto notable cubo de la suma de un binomio y en sustitución de este tema de matemática preuniversitaria hacían uso de concepciones alternativas; asimismo, 6 estudiantes (46\%) en sus respuestas empleaban otros recorridos o procedimientos con respecto a este producto notable que no erran los correctos para concretar la resolución de la integral.

Por último, 4 estudiantes (31\%) no comprendieron que para resolver las integrales se requería de los conocimientos de matemáticas preuniversitarias con respecto a los productos notables, particularmente, el producto de la suma por la diferencia de binomios $\mathrm{y}$, en consecuencia, estos errores son debidos a la comprensión de las instrucciones; de igual forma, 4 estudiantes (31\%), debido a la gran cantidad de información obtenida en la Educación Media General, desarrollaban el cuadrado de un binomio cuando debían desarrollar el cubo de una suma en los productos notables esto debido a sobrecarga cognitiva y costumbres escolares. Cabe destacar que, estos errores de matemática preuniversitaria estuvieron presentes en la resolución de integrales, lo cual repercutió en el buen desenvolvimiento de los estudiantes.

En cuanto a la evaluación parcial, los errores más frecuentes se debieron a concepciones alternativas y de operaciones intelectuales implicadas, con un 54 por ciento de frecuencia, los estudiantes no identificaron la expresión presentada en los integrales haciendo uso de concepciones alternativas propias del educando, llevándolos a través del procedimiento incorrecto empleado a una solución inválida, un ejemplo de ello fue el desarrollo del cuadrado de la diferencia de un binomio, convirtiendo al exponente 2 en un exponente racional y, por el otro, aplicar de forma incorrecta la siguiente propiedad de la potenciación $\left[a^{-n}=\frac{1}{a^{n}}\right]$. 
Con respecto a las operaciones intelectuales implicadas, los estudiantes no realizaron las operaciones de la multiplicación de signos, así como también, al desarrollar los productos notables, para el caso del cuadrado de una diferencia de dos cantidades, no colocaron los signos respectivos al desarrollo del mismo el cual correspondía de forma alterna (positivo - negativo - positivo), así: $\left[(a-b)^{2}=a^{2}-2 a b+b^{2}\right]$. De igual forma hubo errores debidos a la comprensión de las instrucciones 8 por ciento, costumbres escolares o de una mala interpretación 23 por ciento, y de recorridos empleados 31 por ciento. A continuación se presentan estos porcentajes. Todo esto se evidencia en el siguiente Gráfico 2.

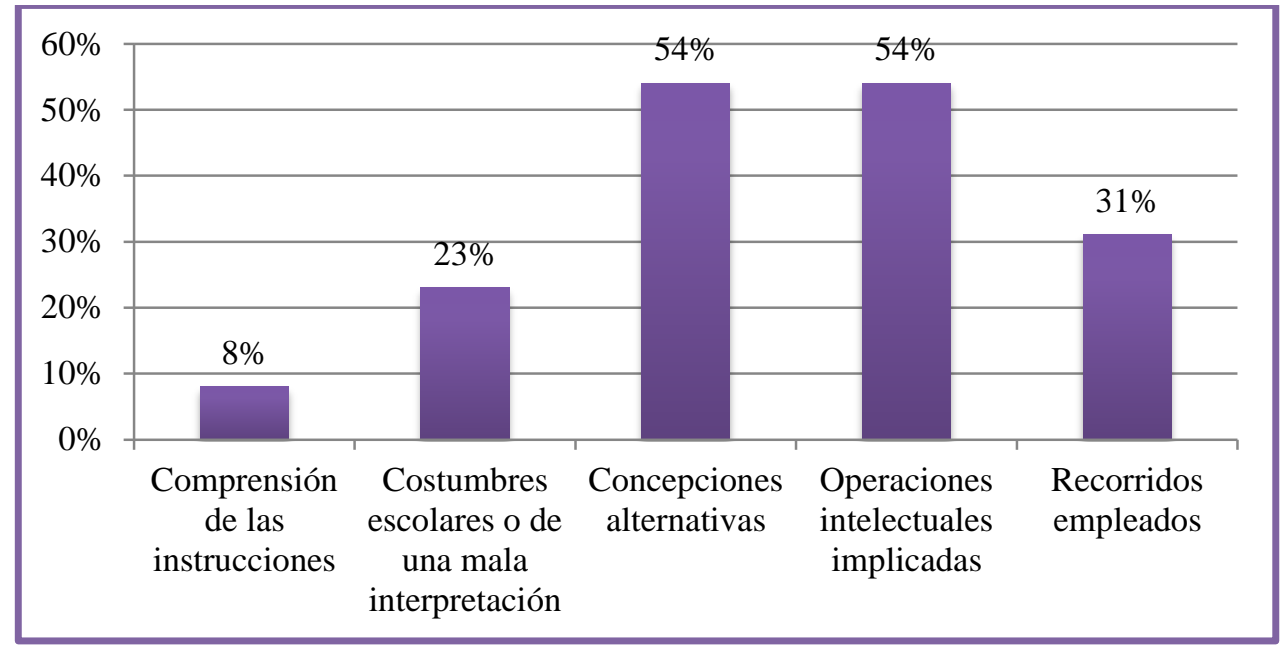

Gráfico 2. Análisis de los errores presentes en la evaluación parcial de acuerdo a la tipología de Astolfi (1999). (Fuente: Terán, 2020)

\section{CONCLUSIONES}

$\mathrm{F}$ inalmente, se llega al cierre de la investigación, se presentan las conclusiones de la investigación con respecto a los objetivos planteados, en el primer objetivo se pudo constatar que los errores presentados fueron de matemática básica (matemática preuniversitaria); sin embargo, se hizo una interpretación de la tipología de errores presentada por Astolfi (1999), donde se pudo diagnosticar que los tipos de errores presentados por los discentes son: debidos a la comprensión de las instrucciones, resultado de los hábitos escolares o de una mala interpretación de las expectativas, como resultado de las 
concepciones alternativas de los alumnos, ligados a las operaciones intelectuales implicadas, errores en los procesos adoptados, debidos a la sobrecarga cognitiva en la actividad.

Una vez que se analizaron las teorías de los tipos de errores, se procedió a dar respuesta al segundo objetivo, el cual permitió determinar los errores presentes en los estudiantes en el desarrollo de las integrales, mediante las pruebas parciales aplicadas en la asignatura Matemática II de Economía Social, es importante considerar al respecto que durante la aplicación de cada uno de los instrumentos se pudo reconocer los tipos de errores presentados con mayor frecuencia, de matemática preuniversitaria, los cuales estaban presentes y evitaban el desenvolvimiento satisfactorio por parte de los estudiantes.

Se pudo constatar que los estudiantes de Matemática II, necesitan de contenidos previos (matemática preuniversitaria) para desarrollar correctamente las integrales en sus diferentes casos, evidenciándose en esta investigación que no poseen estas competencias, lo que trae consigo no aprobar la asignatura, se desmotiven y en un gran porcentaje abandonen la carrera. Por otra parte, en cuanto a las interrogantes formuladas para el presente estudio, es importante considerar que las mismas fueron abordadas, mediante los objetivos específicos, sin embargo a continuación se mencionan y se plantean las respuestas encontradas a lo largo del desarrollo de la presente investigación: ¿Cuáles son los errores en el aprendizaje de matemática preuniversitaria que presentan los estudiantes cursantes de la asignatura matemática II?, el 100\% de los estudiantes presentaron errores con los contenidos de matemática básicos, teniendo mayor énfasis en: propiedades de la potenciación, factorización, conjuntos numéricos y álgebra (manejo de signos de agrupación).

En cuanto a la interrogante ¿Qué errores presentan los estudiantes en la resolución de las integrales propuestas en los parciales respectivos de la asignatura matemática II?, el 100\% de los errores encontrados en los estudiantes se apoyan en la teoría de Astolfi (1999), en cuanto a los procedimientos empleados para la resolución de los ejercicios o planteamientos, la mayoría de los errores prevalecieron en la concepción errada del discente al momento de aplicar un conocimiento de matemática básica en la asignatura de matemática II, las razones por las cuales emplearon los métodos inadecuados variaron según las características de las 
respuestas, bien sea por desconocimiento, por mala interpretación, por comprensión de las instrucciones, entre otros.

Finalmente ¿De qué manera los errores en el aprendizaje de matemática preuniversitaria están presentes en el desempeño de la asignatura Matemática II de Economía Social?, al respecto se pude responder, al haber demostrado que los estudiantes no dominaron las competencias básicas necesarias, lo cual les impidió resolver los planteamientos más complejos que se generaron en la asignatura, de ésta manera se encuentra que en su mayoría los estudiantes presentaron errores por sobrecarga de contenidos, ya que no manejaban adecuadamente la matemática preuniversitaria y al entrar en contacto con nuevas competencias se saturan de contenidos y les impide resolver los ejercicios, ocasionando que no aprueben la materia y en muchos casos el abandono de la carrera, generando así deserción escolar universitaria.

\section{REFERENCIAS}

Astolfi, J. (1999). El error, un medio para enseñar. España: Díada Editora S.L

Briceño, T. (2011). El uso del error en el aprendizaje: Una posible construcción pedagógica argumentativa. Valencia: Universidad de Carabobo

D’Amore, B. (2005). Bases filosóficas, pedagógicas, epistemológicas y conceptuales de la didáctica de la matemática. México: Reverté Ediciones

De la Torre, S. (2004). Aprender de los errores: El tratamiento didáctico de los errores como estrategia de innovación. Buenos Aires, República Argentina: Magisterio del Río de la Plata

Hernández, R. Fernández, C. y Baptista, P. (2010). Metodología de la Investigación. México: McGraw-Hill

Ministerio del Poder Popular para la Educación. (2007). Subsistema de educación secundaria bolivariana, liceos bolivarianos: currículo. Caracas, Venezuela

Ministerio del Poder Popular para la Educación. (2007). Currículo del subsistema de educación primaria bolivariana. Caracas, Venezuela

Mora, D. (2004). Aspectos pedagógicos y didácticos sobre el método de proyectos: Un modelo para su aplicación en educación matemática. En Mora, D. (Ed.). Tópicos en educación matemática. Caracas: Ediciones de la Universidad Central de Venezuela 
Morín, E. (2003). Introducción al pensamiento complejo. Barcelona: Gedisa

Murillo, J. (2003). La investigación sobre eficacia escolar en Iberoamérica. Bogotá: Edición convenio Andrés Bello

Palella, S., y Martins, F., (2012). Metodología de la investigación cuantitativa. Caracas: Fondo editorial de la Universidad Pedagógica Experimental Libertador

Rivas, P. (2005). La educación matemática como factor de deserción escolar y exclusión social. Recuperado de http://www.saber.ula.ve/bitstream/123456789/19954/2/articulo 3.pdf

Socas, M. (2007). Dificultades y Errores en el aprendizaje de las matemáticas. Análisis desde el enfoque lógico semiótico, Revista Dialnet pp. 19-52. Recuperado de http://funes.uniandes. edu.co/1247/ 